\title{
Research Article \\ Effect of Functional Groups in Coal on the Depth of Adsorption Potential Well
}

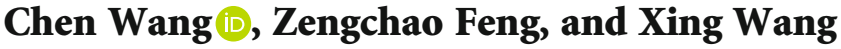 \\ Key Laboratory of In-Situ Property Improving Mining of Ministry of Education, Taiyuan University of Technology, \\ Shanxi Taiyuan 030024, China \\ Correspondence should be addressed to Chen Wang; wangchen_tyut@163.com
}

Received 10 May 2021; Revised 3 July 2021; Accepted 21 July 2021; Published 6 August 2021

Academic Editor: Eloy S. Sanz P rez

Copyright (c) 2021 Chen Wang et al. This is an open access article distributed under the Creative Commons Attribution License, which permits unrestricted use, distribution, and reproduction in any medium, provided the original work is properly cited.

\begin{abstract}
Due to the complexity of the internal structure of natural coal and its characteristic of multicomponent, the depth of its methane adsorption potential well is nonuniform, which makes it difficult to accurately evaluate the adsorption capacity of coal. Besides, in order to find out the factors affecting the depth distribution of potential wells in natural coal, this paper calculated the depth and number of potential wells during methane adsorption in coal according to the Langmuir adsorption kinetics process. Coal samples with different metamorphic degrees were tested and analyzed by infrared spectroscopy diffraction technology. The relationship between the structural parameters of functional groups in coal samples with different metamorphic degrees and the distribution of different depths of adsorption potential wells in coal samples was studied. Some main conclusions are as follows: The number of adsorption potential wells at different depths in natural coal with different metamorphic degrees has multipeak distribution characteristics. With the increase of the metamorphic degree of coal sample, the structures such as aliphatic branched chain structure and oxygen-containing functional groups in coal structure break, fall off, and deoxygenate. The relative content of aliphatic hydrocarbons is significantly reduced and condensed into aromatic hydrocarbons and aromatic ring structures. The different types and quantities of functional groups on the surface of coal samples lead to different forces between coal molecules and methane gas molecules, thus affecting the distribution of different depths of adsorption potential wells in coal samples.
\end{abstract}

\section{Introduction}

An adsorption potential well refers to the position on the surface of coal where the local potential energy is relatively low and methane can be adsorbed, whose depth is related to the mutual attraction between coal and methane [1-3]. Due to the heterogeneity of natural coal $[3,4]$, the difference of oxygen-containing functional groups and side chains on the pore surface of coal [5], and the fractal characteristics of pore surface morphology and other reasons [6,7], the adsorption potential well on coal surface has obvious heterogeneity. Many scholars [8-11] have measured and calculated the potential well depth or the value of adsorption heat when the coal body is adsorbing methane gas by using physical experiments and molecular simulation methods. Results show that the value is concentrated in the range of 0 $30 \mathrm{~kJ} / \mathrm{mol}$. Nie and Duan's calculation [12] shows that the potential well depth on the coal surface is basically consistent with the measured data of adsorption heat. Previous experiments $[13,14]$ have shown that methane adsorption in coal is highly sensitive to temperature variation in the range of $0-270^{\circ} \mathrm{C}$. Therefore, according to the existing research, we can speculate that the potential well depth on the surface of coal has obvious nonuniformity, and there are both deep and shallow depths of potential wells that can adsorb methane on the coal surface.

Structural chemistry of coal has been studied. It is believed that the main body of the coal structure is amorphous polymer highly crosslinked in three-dimensional space and the macromolecular structure of coal is composed of many basic structural units with similar but not identical structures. The core part of the basic structural unit is mainly the condensed directional ring. The periphery of the basic structural unit is connected with three alkyl side chains below carbon and various functional groups. The main functional groups are oxygen-containing functional groups, including 
hydroxyl, carboxyl, methoxy, aldehyde, and carbonyl. Because the atoms of chemical bonds and functional groups remain in a state of continuous vibration, their vibration frequency is similar to that of infrared light. Different chemical bonds or functional groups have different absorption frequencies and will be at different positions in the infrared spectrum. Thus, the information about the chemical bonds or functional groups in the molecule can be obtained. In the midinfrared region of the infrared spectrum, there will be absorption peaks in the wavelength or wavenumber of the absorbed light. The more the wavelength is absorbed, the higher the absorbance, and the stronger the absorption peak. If there are many different absorption peaks in the measured infrared spectrum, it indicates that the sample absorbs many different wavelengths of light. According to the position, strength, and shape of these absorption peaks, the content and distribution of the chemical structure in the sample can be estimated. Besides, different chemical bonds and functional groups in coal structures have different binding abilities with methane molecules. Therefore, in order to analyze the structural reactivity and adsorption performance of coal, it is necessary to comprehensively consider the distribution of aromatic condensation rings, oxygen-containing functional groups, and aliphatic side chains in the coal structure, as well as the variation law of their content with the change of coal metamorphism degree. The influence of functional groups in coal on the adsorption potential well can be explored in this way.

\section{Calculation of Potential Well Depth of Coal Adsorption Methane}

The essence of gas adsorption on the coal pore surface is the result of mutual attraction between molecules on the coal pore surface and gas molecules. The adsorption potential well is an attribute of the coal body, which refers to the adsorption energy when coal surface molecules and adsorbed gas molecules reach the most stable state. It is related to the diversity of coal pore surface morphology and functional groups. The potential well depth characterizes the stability of the adsorption system composed of coal molecules and adsorbed gas molecules. The deeper the potential well is, the greater the adsorption energy is, and the more stable the adsorption system is.

This experiment refers to the temperature range of natural coal reservoirs and the temperature range of coal-bed methane produced by heat injection. The experimental temperature is set between $30^{\circ} \mathrm{C}$ and $150^{\circ} \mathrm{C}$, and the temperature interval is $20^{\circ} \mathrm{C}$. There are 7 groups of isothermal conditions in total. Under each isothermal condition, referring to the gas pressure of natural coal reservoirs, a total of 11 adsorption pressure measuring points with a pressure interval of $0.2 \mathrm{MPa}$ were set within the range of $0.2 \mathrm{MPa} \sim 2.2 \mathrm{MPa}$ to carry out the isothermal adsorption experiments. According to the Langmuir equation based on adsorption kinetics, the adsorption capacity of different adsorption pressure sections and the corresponding adsorption pressure were calculated and analyzed by piecewise fitting. The pressure measuring points of each fitting pressure section were 3 , and a total of
9 pressure sections were selected. When fitting the data, the Langmuir equation is deformed as follows:

$$
\frac{1}{n}=\frac{1}{a b p}+\frac{1}{a} .
$$

In Formula (1), $n$ is methane adsorption capacity in coal (mol) and $a$ represents the total number of methane adsorption sites on the surface (mol), which is related to the surface area of coal. It does not change with temperature or adsorption pressure; $p$ is isothermal adsorption pressure (MPa); $b$ is the ratio of adsorption rate to desorption rate of methane molecules on the coal surface in unit time; the expression is [15-17]

$$
b=b_{m} e^{(\varepsilon / k T)} .
$$

In formula (2), $b_{m}$ is a proportional constant.

According to formula (1), the adsorption pressure $p$ and the corresponding adsorption quantity $n$ in nine pressure sections selected under each isothermal condition were fitted and calculated, and the total potential well $a$ and the rate parameter $b$ of methane adsorption in coal under each pressure section were obtained. The proportional constant $b_{m}$ in formula (2) should be calculated when calculating the average potential well depth of the coal body under each pressure section. Since the dependence of $b_{m}$ on temperature is negligible, the results can be calculated according to formula (2) by fitting the $b$ values of seven groups of different isothermal adsorption and the corresponding absolute temperature values. Finally, $b_{m}$ of the anthracite coal sample is 0.0075 , that of the lean coal sample is 0.0196 , that of the coking coal sample is 0.0294 , and that of the lignite sample is 0.0329 . This result can be used to calculate the average potential well depth of methane gas adsorbed by four natural coal bodies under each pressure section.

The $b$ value of methane adsorption rate parameters of coal samples at different pressure sections under each isothermal condition was obtained by $b_{m}$ value calculation and fitting. According to formula (2), the average well depth $\varepsilon_{c, T j}$ corresponding to each pressure section is calculated. Finally, according to formulas (3) and (4), for different potential well depths $\varepsilon_{i}$ and the corresponding number of potential wells $N_{i}$,

$$
\begin{gathered}
\varepsilon_{i}=\frac{\varepsilon_{c+1, T j} \times a_{c+1, T j}-\varepsilon_{c, T j} \times a_{c, T j}}{a_{c+1, T j}-a_{c, T j}}, \\
N_{i}=a_{c+1, T j}-a_{c, T j} .
\end{gathered}
$$

In the formula, $\varepsilon_{c, T j}$ is the average potential well depth $(\mathrm{kJ} / \mathrm{mol})$ of methane adsorption on the coal body in $c$ pressure section at $T_{j}$ temperature, $a_{c, T j}$ is the total amount of potential well (mol) of methane gas adsorbed by coal in $c$ pressure section at $T_{j}$ temperature, $\varepsilon_{i}$ is the potential well depth $(\mathrm{kJ} / \mathrm{mol})$ of methane adsorption in coal from $c$ pressure section to $c+1$ pressure section, and $N_{i}$ is the number of potential wells (mol) with a potential well depth of $\varepsilon_{i}$. 
Through the statistical analysis of the calculation results of the potential well depth and the number of potential wells, the columnar diagram of the relationship between the potential well depth and the number of potential wells for methane adsorption on four coal samples can be obtained, as shown in Figure 1. The distribution proportion of potential well depth in coal samples with different metamorphic degrees is counted, and the results are shown in Table 1.

\section{Infrared Spectrum Experiment}

3.1. Coal Sample Preparation. In this paper, four kinds of coal samples with different ranks were selected for infrared spectroscopy experiments, namely, anthracite, lean coal, coking coal, and lignite. Samples of each coal rank were selected from typical coal-rich areas in North China and Northeast China. Anthracite was taken from 9\# coal seam of Sihe Coal Mine in Jincheng City, Shanxi Province; lean coal was taken from 3\# coal seam of Sanyuan Zhongneng Coal Mine in Changzhi City, Shanxi Province; coking coal was taken from 8\# coal seam of Malan Coal Mine in Taiyuan City, Shanxi Province; and lignite was taken from II\# coal seam group of Lingdong Coal Mine in Manzhouli City, Inner Mongolia. The coal samples used in this section are 200 mesh (particle size less than $0.074 \mathrm{~mm}$ ) powder coal samples, which are obtained by grinding after the potential well depth measurement experiment of each sample.

3.2. Experimental Method. The VERTEX 80v infrared spectrometer produced by German Beuker Company in the Mineral Processing Laboratory of Taiyuan University of Technology is used to test coal samples with different metamorphic degrees. The experimental equipment is shown in Figure 2. The test steps are as follows: (1) The dried coal samples are placed in an agate mortar and thoroughly ground. (2) The high-purity potassium bromide (KBr) was about $100 \mathrm{mg}$, and a small amount of coal sample was added. The mass ratio of coal sample to potassium bromide was $1: 100$, and the mixture was fully ground to 200 mesh. (3) The mixed samples are loaded into the mold of the sample holder. The mold is pressed to $100000 \mathrm{~N} / \mathrm{cm}^{2}$ by the tablet press for $1 \mathrm{~min}$ to ensure that the formed sample sheet is uniform and there is no damage or leakage. (4) After the infrared spectrometer is preheated, the measurement background is scanned, and the sample frame of the pressed coal sample sheet is placed in the sample room of the infrared spectrometer. The instrument scanned 16 times in total, and the infrared spectrum is obtained after the baseline correction.

\section{Experimental Result}

4.1. Conventional Characteristics of Coal Samples. Before the quantitative analysis of infrared spectral parameters, the elemental analysis of each coal sample should be carried out according to GB/T476-2001. The analysis results are shown in Table 2. The distribution range of four samples on the whole vitrinite reflectance $R_{o}$ max ranges from $0.4 \%$ to $3.1 \%$, and the corresponding coal rank ranges from lignite to anthracite. It can be clearly seen that although the coal sam- ples have different metamorphic degrees, all the characteristic adsorption bands are similar, indicating that these samples have similar surface structures and functional groups [18].

4.2. Infrared Spectral Characteristics of Coal Samples. Infrared spectra of samples with different coal ranks were obtained after calibrating the data by baseline, as shown in Figure 3. The infrared spectra of each sample were analyzed by peak fitting, and the absorption peak of the infrared spectrum was assigned according to the previous studies [19]. The whole infrared spectra were divided into four parts: the aromatic structure whose infrared wave number is between 700 and $900 \mathrm{~cm}^{-1}$, the oxygen-containing functional group between 1000 and $1800 \mathrm{~cm}^{-1}$, the aliphatic branched chain between 2800 and $3000 \mathrm{~cm}^{-1}$, and the hydroxyl functional group between 3000 and $3600 \mathrm{~cm}^{-1}$. As shown in Figure 4, taking anthracite samples as an example to carry out peak fitting, the fitting correlation coefficients are all greater than 0.99 , and the peak distribution follows previous studies [20-22].

\subsubsection{Aromatic Structure Parameters}

(1) Aromatic Carbon Ratio. Apparent aromatic carbon rate $f_{a}$ of coal samples is used to characterize the content of aromatic carbon. According to previous studies [23, 24], only aromatic and aliphatic carbon atoms are included in all types of carbon atoms. Formulas (5) to (7) can be used to calculate $f_{a}$ :

$$
\begin{aligned}
& \frac{H_{\mathrm{al}}}{H}=\frac{H_{\mathrm{al}}}{H_{\mathrm{al}}+H_{\mathrm{ar}}}=\frac{A_{2800-3000}}{A_{2800-3000}+A_{700-900}}, \\
& \frac{C_{\mathrm{al}}}{C}=\frac{H_{\mathrm{al}} / H \times H / C}{H_{\mathrm{al}} / C_{\mathrm{al}}}, \\
& f_{a}=1-\frac{C_{\mathrm{al}}}{C} \text {. }
\end{aligned}
$$

The value of $H_{\mathrm{al}} / H$ is the ratio of hydrogen content in fat structure $\left(H_{\mathrm{al}}\right)$ to total hydrogen atom $(H)$. One is determined by the area of the integral absorption band at 2800$3000 \mathrm{~cm}^{-1}\left(A_{2800-3000}\right)$, and the other is determined by the area of the integral absorption band at $700-900 \mathrm{~cm}^{-1}$ $\left(A_{700-900}\right) . C_{\mathrm{al}} / C$ represents the percentage of aliphatic carbon to total carbon; $H / C$ is the ratio of hydrogen to carbon calculated from elemental analysis, as shown in Table $2 . H_{\mathrm{al}} / \mathrm{C}_{\mathrm{al}}$ is the number ratio of hydrogen and carbon atoms in an aliphatic structure. For coal, the value is $1.8[25,26]$, which is the proportion of hydrogen and carbon in aliphatic groups. Thus, the relationship between the aromatic carbon ratios of different coal samples and the metamorphic degree of coal samples can be obtained, as shown in Figure 5. According to the calculation statistics, the aromatic carbon rate of coal samples with a high metamorphic degree is significantly higher than that of coal samples with a medium and low metamorphic degree. With the increase of coal metamorphism degree, the value of aromatic carbon ratio showed an increasing trend, indicating that the proportion of aromatic 


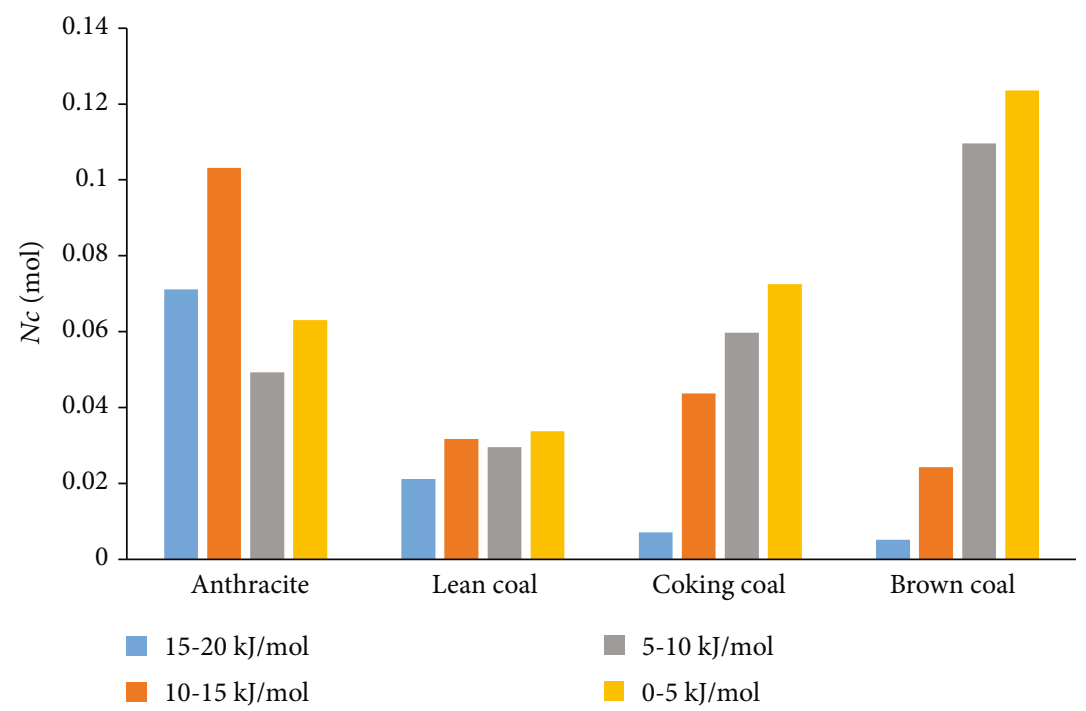

FIGURE 1: Relationship between potential well depth and number of potential wells for methane adsorption on coal samples.

TABle 1: Statistics of potential well depth distribution in different metamorphic coal samples.

\begin{tabular}{|c|c|c|c|c|c|}
\hline \multirow{2}{*}{ Types of coal samples } & \multirow{2}{*}{$R_{o \text { max }}(\%)$} & \multicolumn{4}{|c|}{ Distribution ratio of potential well depth (\%) } \\
\hline & & $15 \sim 20 \mathrm{~kJ} / \mathrm{mol}$ & $10 \sim 15 \mathrm{~kJ} / \mathrm{mol}$ & $5 \sim 10 \mathrm{~kJ} / \mathrm{mol}$ & $0 \sim 5 \mathrm{~kJ} / \mathrm{mol}$ \\
\hline Anthracite & 3.1 & 24.84 & 35.98 & 17.19 & 22.00 \\
\hline Lean coal & 2.1 & 18.19 & 27.29 & 25.43 & 29.10 \\
\hline Coking coal & 1.3 & 3.88 & 23.88 & 32.62 & 39.62 \\
\hline Brown coal & 0.4 & 1.94 & 9.26 & 41.74 & 47.06 \\
\hline
\end{tabular}

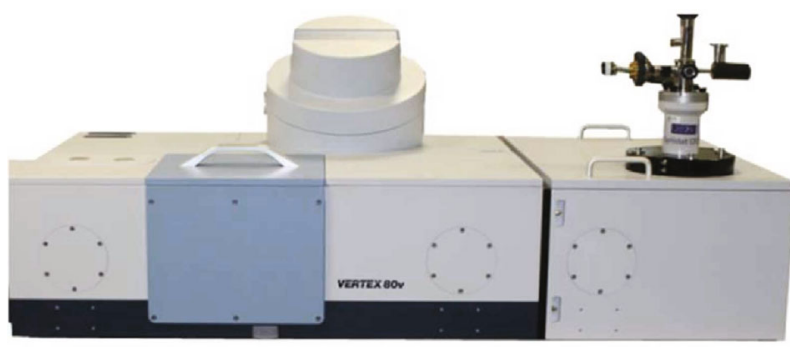

FigURE 2: VERTEX 80v infrared spectrum analyzer.

TABLE 2: Elemental analysis and maceral determination parameters of coal samples.

\begin{tabular}{lcccccc}
\hline $\begin{array}{l}\text { Types of coal } \\
\text { samples }\end{array}$ & $\begin{array}{c}\mathrm{C} \\
(\%)\end{array}$ & $\begin{array}{c}\mathrm{H} \\
(\%)\end{array}$ & $\begin{array}{c}\mathrm{O} \\
(\%)\end{array}$ & $\begin{array}{c}\mathrm{N} \\
(\%)\end{array}$ & $\begin{array}{c}\mathrm{S} \\
(\%)\end{array}$ & $\begin{array}{c}R_{o} \max \\
(\%)\end{array}$ \\
\hline Anthracite & 86.52 & 2.64 & 6.83 & 0.69 & 3.32 & 3.1 \\
Lean coal & 88.41 & 3.49 & 5.22 & 1.15 & 1.70 & 2.1 \\
Coking coal & 86.65 & 4.39 & 5.27 & 1.30 & 2.39 & 1.3 \\
Brown coal & 74.19 & 4.29 & 19.08 & 1.25 & 1.19 & 0.4 \\
\hline
\end{tabular}

carbon content in coal samples gradually increased, and other functional groups and aliphatic side chains fell off from the coal structure.

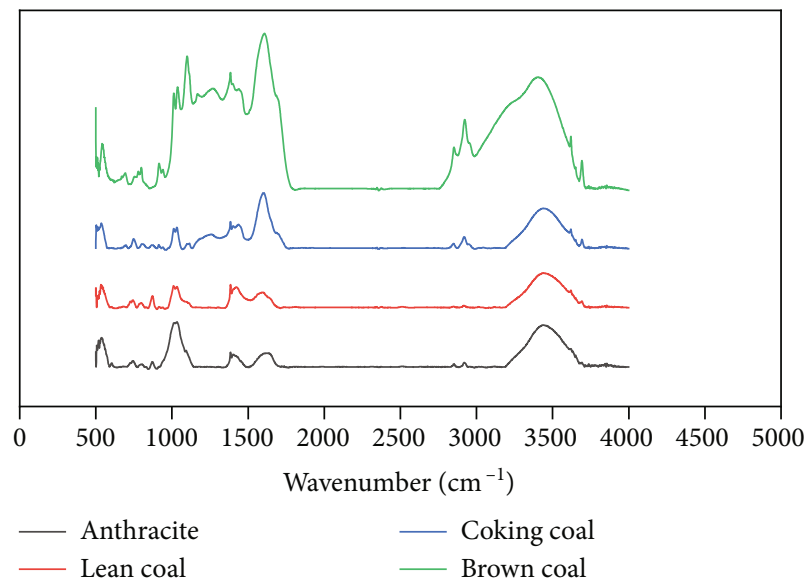

FIGURE 3: Infrared spectra of four coal samples after calibration.

(2) Aromaticity. Parameter AR is another indicator of aromaticity and rank of coal metamorphism. It can be used to compare the relative abundance of aromatic and aliphatic functional groups. It is represented by the ratio of out-ofplane deformation vibration intensity of substituted aromatic hydrocarbons near $700-900 \mathrm{~cm}^{-1}$ to total aliphatic $\mathrm{CH}$ 


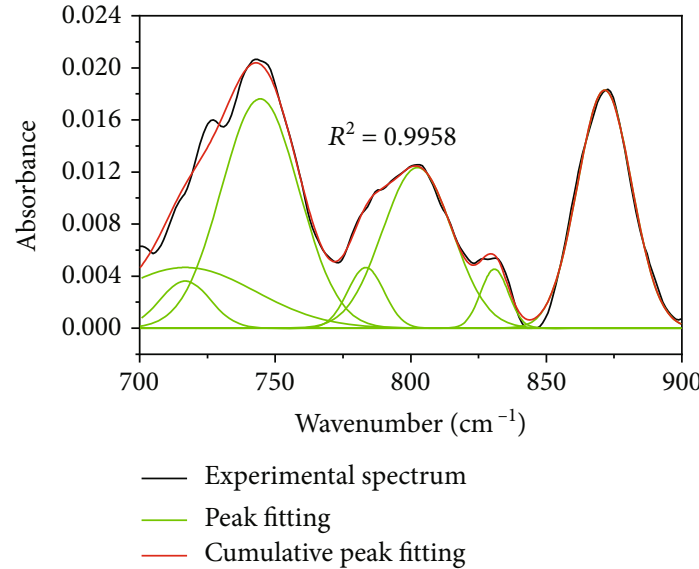

(a)

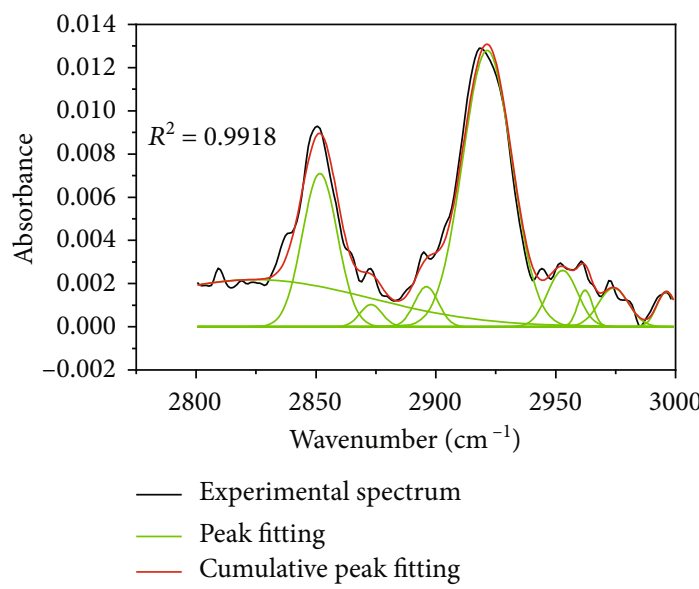

(c)

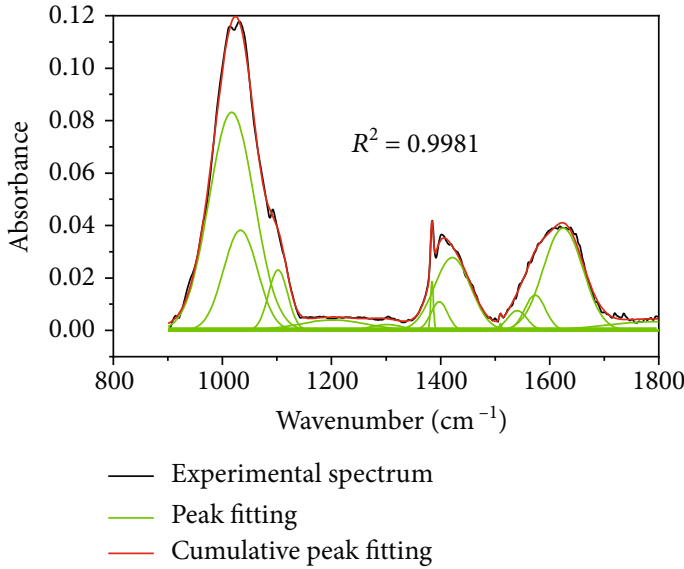

(b)

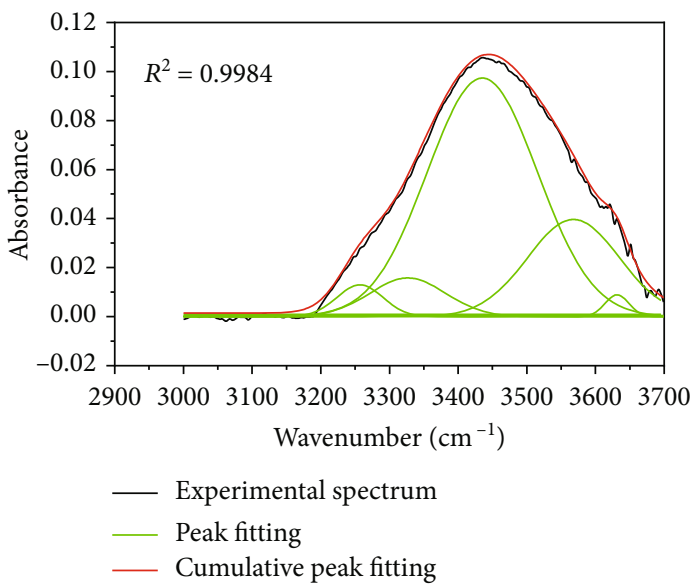

(d)

FIGURE 4: Fitting curve of infrared spectrum of functional groups of anthracite.

content near $2800-3000 \mathrm{~cm}^{-1}$ [27]:

$$
\mathrm{AR}=\frac{A_{700-900}}{A_{2800-3000}}
$$

In addition to the aromaticity of infrared parameters, AR index parameters were used to characterize the aromaticity of different coal samples and the rank of coal metamorphism degree. The statistical analysis results of the AR index are shown in Figure 6. It can be found that there is a positive correlation between aromatic AR index and coal sample metamorphic grade index (maximum vitrinite reflectance $R_{o}$ max ). The aromaticity of the coal sample increases with the increase of the coal sample metamorphic grade, which is caused by the continuous shedding of the fat chain during coal formation.

(3) Condensation Degree of Aromatic Structure. The intensity of the deformed vibration band on the outer surface of aromatic hydrocarbon $(\mathrm{CH})$ is related to the peak area of the $\mathrm{C}$ $=\mathrm{C}$ stretching vibration band on the aromatic ring. The intensity ratio between the two is the degree of aromatic

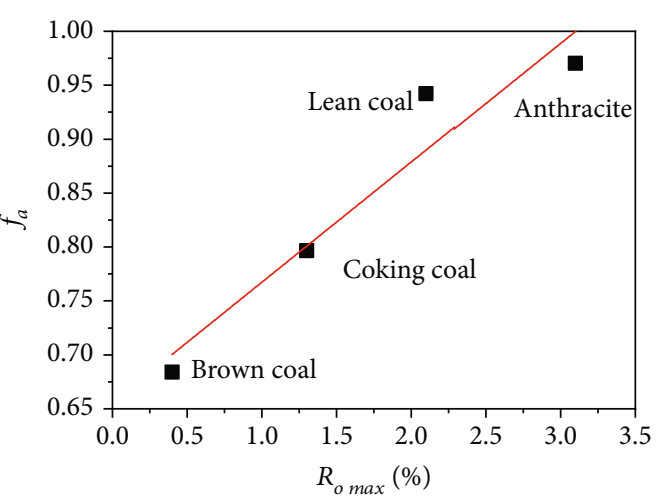

FIGURE 5: Relationship between aromatic carbon ratio of infrared parameters and metamorphic degree of coal samples.

structure condensation (DOC) $[24,27]$ :

$$
\text { DOC }=\frac{A_{700-900}}{A_{1600}} .
$$

The relationship between aromatic structure condensation degree (DOC) and metamorphism degree index of coal 


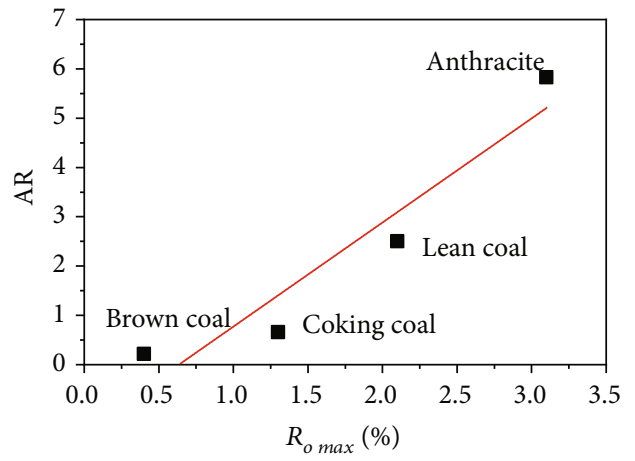

FIGURE 6: Relationship between aromaticity of infrared parameters and metamorphic degree of coal samples.

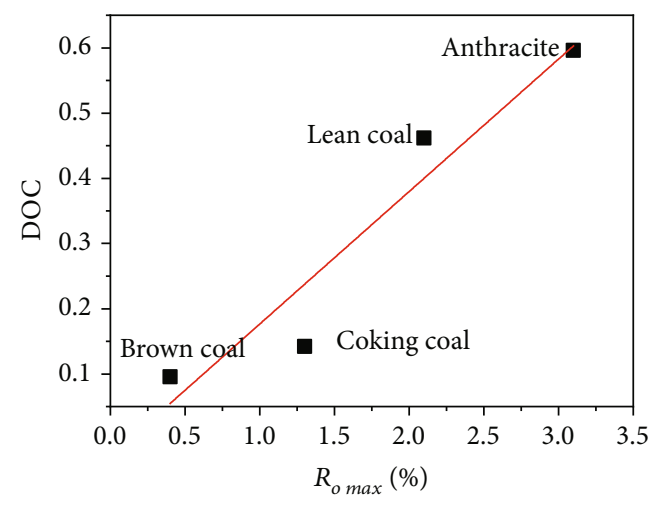

FIgURE 7: Relationship between aromatic condensation degree of infrared parameters and metamorphic degree of coal samples.

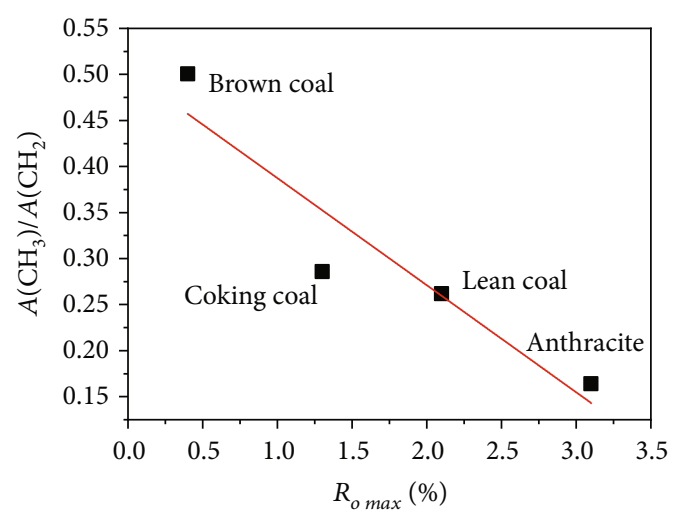

FIGURE 8: Relationship between infrared parameters of fat structure parameters and coal metamorphism.

sample (maximum vitrinite reflectance $R_{o \text { max }}$ ) is shown in Figure 7, which clearly shows that with the increase of the coal sample metamorphism degree (i.e., the increase of $R_{o \max }$ ), the aromatic ring structure condensation degree (DOC) also increases.

4.2.2. Fat Structure Parameters. The ratio of methyl to methylene strength $\left(A\left(\mathrm{CH}_{3}\right) / A\left(\mathrm{CH}_{2}\right)\right)$ in coal was used to calcu-

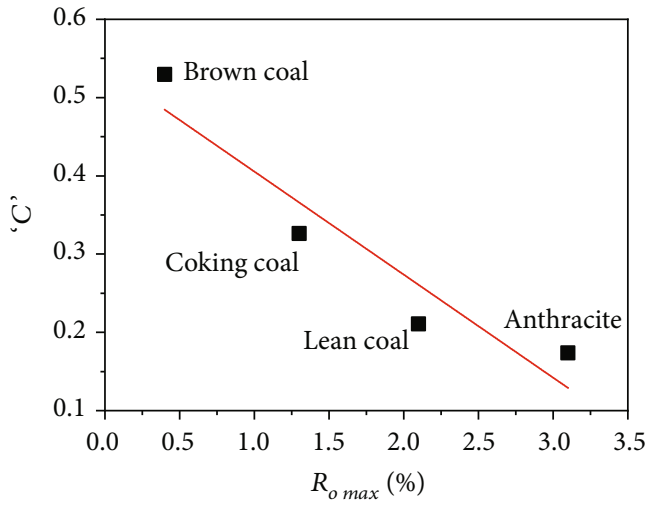

FIgURE 9: Relationship between infrared parameter ' $C$ ' index and coal sample metamorphism.

late the length and the branching degree of the fat side chain. The higher the value of $A\left(\mathrm{CH}_{3}\right) / A\left(\mathrm{CH}_{2}\right)$, the longer the aliphatic side chain corresponding to the aromatic ring, while the lower the value, the more compact the structure is and the smaller the space between aromatic clusters [25, 28]. The strength ratio of methyl to methylene is

$$
\frac{A\left(\mathrm{CH}_{3}\right)}{A\left(\mathrm{CH}_{2}\right)}=\frac{A_{2950}}{A_{2920}} \text {. }
$$

In the formula, $A_{2920}$ and $A_{2950}$ are, respectively, attributed to the asymmetric tensile strength of methylene and methyl groups.

The relationship between the ratio of methyl to methylene strength $\left(A\left(\mathrm{CH}_{3}\right) / A\left(\mathrm{CH}_{2}\right)\right)$ and the metamorphic index (maximum vitrinite reflectance $R_{o \text { max }}$ ) of coal samples is shown in Figure 8. The larger the parameter value is, the longer the length of the side chain in the coal is, and the less the branched chain is. It can be seen from the diagram that with the increase of coal sample metamorphism, the fat structure parameters gradually decrease, and the length and branching degree of fat structure in coal gradually decrease. $\mathrm{Li}$ [22], Li [29], Zou [30], and others also found this relationship between the two. This shows that in the process of coal formation, the aliphatic side chain structure is constantly shedding, the methylene condensation is formed into aromatic rings, and the aromaticity of coal increases.

4.3. Structural Parameters of Oxygen-Containing Groups. The structural parameter ' $C$ ' of oxygen-containing functional groups represents the change of the ratio of $\mathrm{C}=\mathrm{O}$ and $\mathrm{C}=\mathrm{C}$ groups of carboxyl and carbonyl groups in the coal structure, which is used to characterize the maturity of coal. ' $C$ ' is determined by formula (11) [28, 31]:

$$
{ }^{\prime} C^{\prime}=\frac{A_{1650-1800}}{A_{1650-1800}+A_{1600}} .
$$

Figure 9 shows the variation trend of the $\mathrm{C}=\mathrm{O}$ group relative to the metamorphism index of the coal sample (maximum vitrinite reflectance $R_{o} \max$ ). Through Figure 9 , it can 

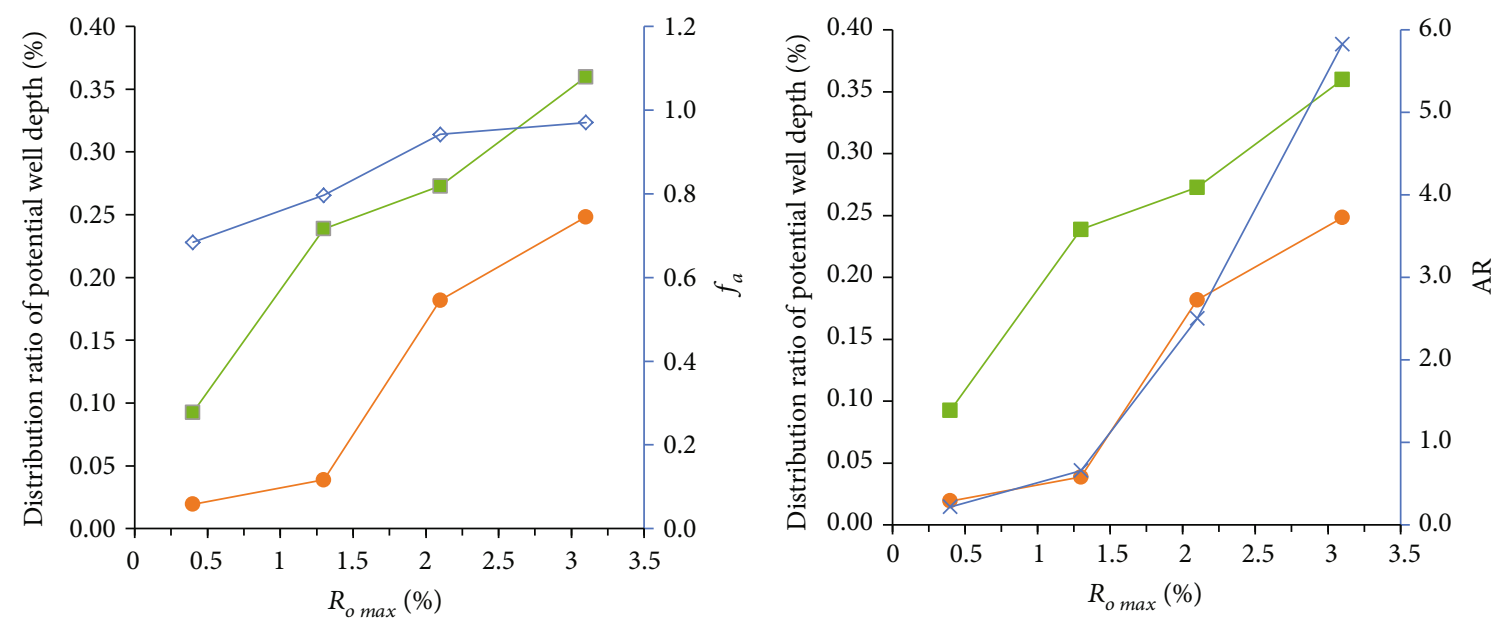

$$
\begin{aligned}
& \longrightarrow 15 \sim 20 \mathrm{~kJ} / \mathrm{mol} \\
& -10 \sim 15 \mathrm{~kJ} / \mathrm{mol} \\
& \smile f_{a}
\end{aligned}
$$

$$
\begin{aligned}
& -15 \sim 20 \mathrm{~kJ} / \mathrm{mol} \\
& -\quad 10 \sim 15 \mathrm{~kJ} / \mathrm{mol} \\
& \rightarrow \mathrm{AR}
\end{aligned}
$$

(a)
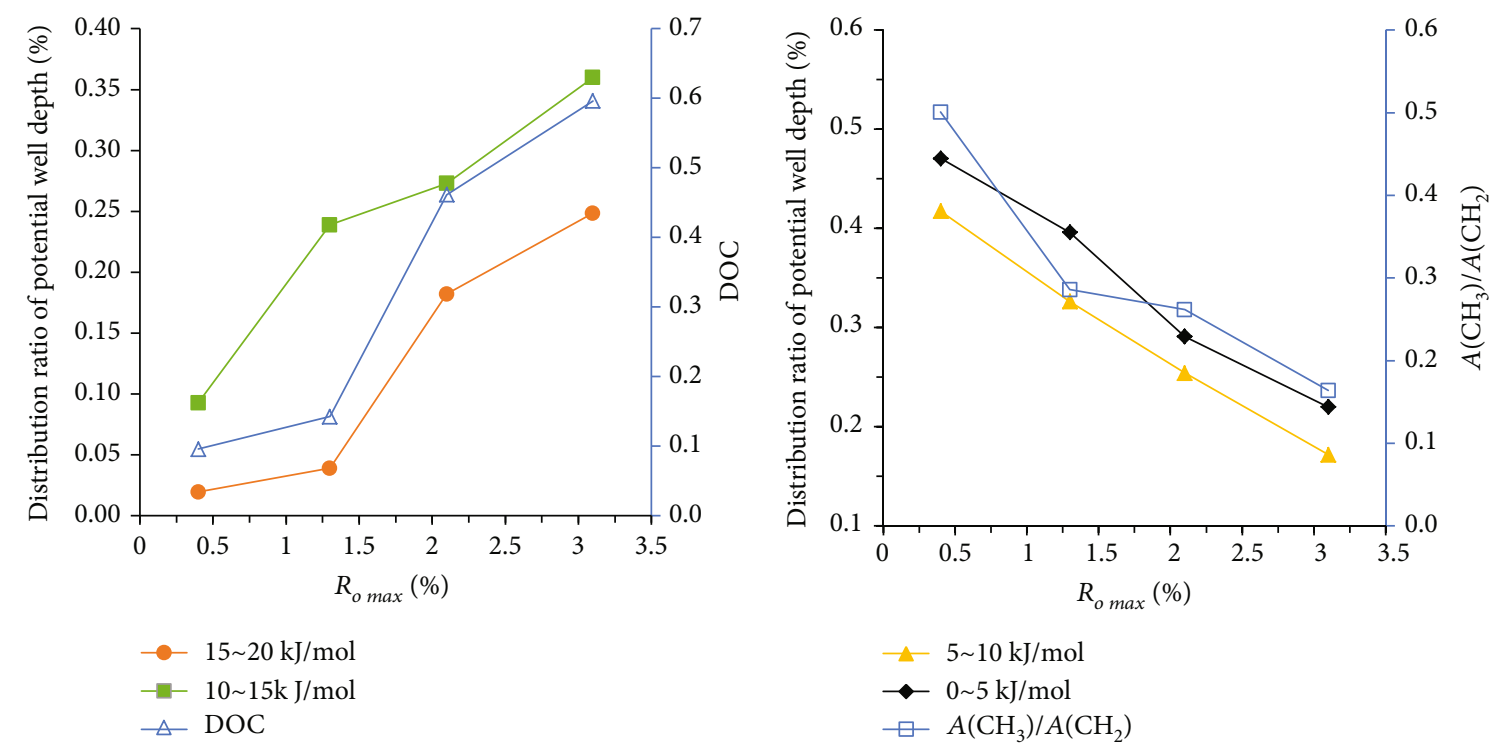

(c)

(d)

Figure 10: Continued. 


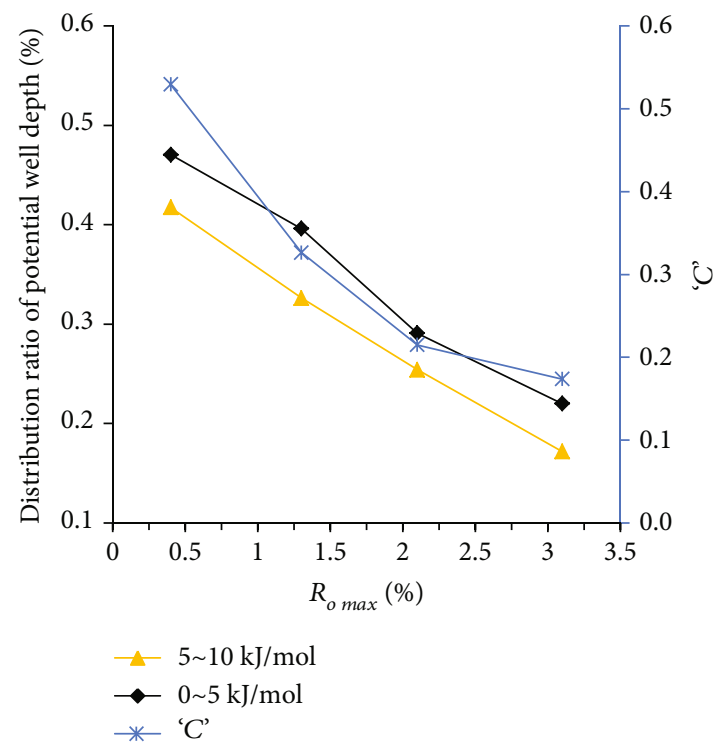

(e)

FIGURE 10: The relationship between infrared spectrum diffraction parameters and potential well depth distribution.

be found that there is a negative correlation between the two, and the ' $C$ ' index decreases rapidly first and then gradually stabilizes with the increase of the coal metamorphism degree. Attention should be paid to the lowest point of the ' $C$ ' index near $R_{o \text { max }}=2.0 \%$, which means that with the increase of the maximum vitrinite reflectance $R_{o \text { max }}$, when $R_{o \text { max }}<2.0 \%$, the $\mathrm{C}=\mathrm{O}$ group in coal decreases rapidly and then decreases slowly until it becomes stable when $R_{o \text { max }}>2.0 \%$. This is because, after the first coal-forming change, there are still a small amount of oxygen-containing functional groups containing $\mathrm{C}=\mathrm{O}$ groups such as carbonyl $(-\mathrm{C}=\mathrm{O})$ and carboxyl $(-\mathrm{COOH})$ in the coal structure, which are falling off in the process of coal-forming, and then, the maturity of the coal is continuously improved. However, before the asphalteneization, a large number of oxygen-containing functional groups such as carboxyl $(-\mathrm{COOH})$ in the coal have already been shed, so the remaining small amount of carboxyl $(-\mathrm{COOH})$ will shed slowly in the subsequent asphalteneization. The relationship between the two shows that the coalification process is the deoxidation process in which the $\mathrm{C}=\mathrm{O}$ group in coal is gradually transformed into the $\mathrm{C}=\mathrm{C}$ structure. The $\mathrm{C}=\mathrm{O}$ functional group is gradually broken, deoxygenated, and condensed into an aromatic ring structure. Therefore, the oxygen-containing functional groups in coal with high metamorphism degrees are significantly less than those in coal with low metamorphism degrees.

\section{Analysis and Discussion}

The adsorption of methane gas by coal samples is caused by "van der Waals" force between adsorbate and adsorbent molecules. The different types and quantities of functional groups on the surface of coal samples lead to different forces between coal samples and methane gas molecules, which further affects the distribution of different depths of adsorption potential wells in coal samples. The greater the molecular polarity of the functional groups on the surface of the coal sample is, the higher the force between the functional groups and the methane molecules, the deeper the adsorption potential well of the coal sample for the methane molecules, and the stronger the stability of the coal sample for the adsorption of the methane molecules.

The distribution of adsorption potential well of coal samples with different metamorphic degrees is closely related to the types and quantities of functional groups on the coal surface. Figure 10 shows the relationship between the structural parameters of infrared spectrum diffraction of coal samples with different metamorphic grades and the distribution of potential wells at different depths.

From Figures 10(a)-10(c), it can be seen that the aromaticity ratio, aromaticity, and condensation parameters of the coal sample structure show varying degrees of growth with the increase of coal sample metamorphism. This is consistent with the variation law of the distribution ratio of the adsorption potential well in the range of $10-15 \mathrm{~kJ} / \mathrm{mol}$ and $15-$ $20 \mathrm{~kJ} / \mathrm{mol}$ with the metamorphic degree of coal sample, indicating that the aromatic carbon rate, aromaticity, and condensation degree parameters of the coal sample structure play a key role in the distribution ratio of the relatively high depth of adsorption potential wells. This shows that with the increase of the metamorphism degree of the coal sample, the degree of aromatization of the coal molecular structure increases, and the relative content of aliphatic functional groups decreases. Besides, the degree of condensation of the aromatic ring also increases, because in the process of coalification from low metamorphism to high metamorphism, oxygen-containing functional groups such as hydroxyl group and carboxyl group fall off [31]. As shown in Figures 10(a)10 (c), when the maximum vitrinite reflectance $R_{o}$ max is in the range of $1.4 \% \sim 2.1 \%$, the aromatic-carbon ratio, 
aromaticity, and degree of condensation of the coal sample structure have the greatest changes [32]. It can be seen from Figures $10(\mathrm{~d})$ and $10(\mathrm{e})$ that the length of the fat side chain and the ' $C$ ' index parameters of the coal sample structure show different degrees of decrease with the increase of the coal sample metamorphism degree. This is consistent with the variation law of the distribution ratio of adsorption potential wells in the range of $0-5 \mathrm{~kJ} / \mathrm{mol}$ and $5-10 \mathrm{~kJ} / \mathrm{mol}$ with the metamorphism of coal sample, indicating that the length of the fat side chain of coal sample structure and the structural parameter ' $C$ ' of oxygen-containing functional groups play a key role in the distribution ratio of low and medium depths of adsorption potential wells. It shows that with the progress of coalification, the maturity of organic matter gradually increases, and the structure of the fat chain in the coal structure becomes shorter. In the process, the content of the methylene $\left(-\mathrm{CH}_{2}\right)$ side chain gradually decreases; the structure of aliphatic hydrocarbons gradually falls off, condensed into aromatic ring structures and other macromolecular structures; and the aromaticity shows an increasing trend [32-35].

\section{Conclusions}

In this paper, the number distribution of different depths of adsorption potential wells in natural coal with different ranks is calculated statistically. Four coal samples with different metamorphic degrees are tested and analyzed by infrared spectrum diffraction technology. The relationship between the structural parameters of functional groups of coal samples with different metamorphic degrees and the distribution of different depths of adsorption potential wells in coal samples is studied. The conclusions are as follows:

(1) Through the statistical analysis of the calculation results of the potential well depth and the number of potential wells, the distribution diagram of the potential well depth and the number of potential wells of methane gas adsorbed by coal is obtained. Due to the diversity of natural coal components and the complexity of pore and fracture structure, the number of potential wells at different depths in natural coal with different metamorphic degrees has multipeak distribution characteristics. The distribution of adsorption potential well depth in coal samples shows different trends with the change of metamorphic degree

(2) With the increase of coal sample metamorphism degree, the aromatic carbon rate $f_{a}$, aromatic AR, and aromatic structure condensation degree DOC of coal all showed increasing trends, and the fat structural parameters and the structural parameters ' $C$ ' of the oxygen-containing functional groups showed decreasing trends. The structure of the aliphatic branched chain and oxygen-containing functional groups in the coal structure breaks, falls off, and deoxygenates. The relative content of aliphatic hydrocarbons is significantly reduced and condensed into aromatic hydrocarbons and aromatic rings

(3) The different types and quantities of functional groups on the surface of coal samples lead to different forces between coal samples and methane gas molecules, thus affecting the distribution of different depths of methane adsorption potential wells in coal samples. The aromatic carbon rate $f_{a}$, aromatic AR, and aromatic structure condensation parameter DOC of the coal sample structure play a key role in the adsorption potential well at a relatively high depth of $10-15 \mathrm{~kJ} / \mathrm{mol}$ and $15-20 \mathrm{~kJ} / \mathrm{mol}$. The variation law of these three infrared spectral structure parameters with the metamorphic degree of the coal sample is consistent with the variation law of the distribution ratio of adsorption potential well at a relatively high depth with the metamorphic degree of coal sample. The length of the aliphatic side chain of the coal sample structure and the structural parameters ' $C$ ' of the oxygen-containing functional groups play a key role in the adsorption potential wells at the relatively low depth of $0-5 \mathrm{~kJ} / \mathrm{mol}$ and at the medium depth of $5-10 \mathrm{~kJ} / \mathrm{mol}$. The variation law of the two infrared spectral structural parameters with the metamorphic degree of the coal sample is consistent with the variation law of the distribution ratio of the adsorption potential wells at the low and medium depths with the variation of the metamorphic degree of the coal sample

\section{Data Availability}

The raw/processed data required to reproduce these findings cannot be shared at this time as the data also forms part of an ongoing study.

\section{Conflicts of Interest}

The authors declare that there is no conflict of interest regarding the publication of this paper.

\section{Acknowledgments}

The authors would like to thank the National Natural Science Foundation of China (nos. 21373146) for financial assistance, and we thank Master Sun for polishing this manuscript.

\section{References}

[1] X. Li, X. H. Fu, A. H. Liu et al., "Methane adsorption characteristics and adsorbed gas content of low-rank coal in China," Energy \&fuels, vol. 30, no. 5, pp. 3840-3848, 2016.

[2] Z. X. Liu and Z. C. Feng, "Theoretical study on adsorption heat of methane in coal," Journal of China Coal Society, vol. 37, no. 4, pp. 647-653, 2012.

[3] L. Ji, T. Zhang, L. Kitty, J. Qu, and X. Zhang, "Experimental investigation of main controls to methane adsorption in clayrich rocks," Applied Geochemistry, vol. 27, no. 12, pp. 25332545, 2012. 
[4] H. P. Wang, Y. S. Yang, Y. D. Wang, J. L. Yang, J. Jia, and Y. H. Nie, "Data-constrained modelling of an anthracite coal physical structure with multi-spectrum synchrotron X-ray CT," Fuel, vol. 106, no. 2013, pp. 219-225, 2013.

[5] F. B. Zhou, S. Liu, Y. Pang, J. Li, and H. Xin, "Effects of coal functional groups on adsorption microheat of coal bed methane," Energy \& Fuels, vol. 29, no. 3, pp. 1550-1557, 2015.

[6] X. Y. Men, S. Tao, Z. X. Liu, W. Tian, and S. Chen, "Experimental study on gas mass transfer process in a heterogeneous coal reservoir," Fuel Processing Technology, vol. 30, no. 3, pp. 437-442, 2013.

[7] S. Tao, S. D. Chen, D. Z. Tang, X. Zhao, H. Xu, and S. Li, "Material composition, pore structure and adsorption capacity of low-rank coals around the first coalification jump: a case of eastern Junggar Basin, China," Fuel, vol. 211, no. 2018, pp. 804-815, 2018.

[8] W. P. Jiang, "Microscopic mechanism study on the influence of coal rank on adsorption capacity," China Coalbed Methane, vol. 6, no. 2, pp. 19-22, 2009.

[9] A. Nodzeriski, "Sorption and desorption of gases $\left(\mathrm{CH}_{4}, \mathrm{CO}_{2}\right)$ on hard coal and active carbon at elevated pressures," Fuel, vol. 77, no. 11, pp. 1243-1246, 1998.

[10] C. Zhang, X. S. Lu, and A. Z. Gu, "The adsorption heat research status of natural gas and hydrogen adsorption storage," Acta Energiae Solaris Sinica, vol. 25, no. 2, pp. 249-253, 2004.

[11] L. Guo and Z. S. Wu, "Mechanism of methane's adsorption on nanometer active carbon at supercritical state," Chinese Journal of Chemical Physics, vol. 24, no. 5, pp. 737-742, 2008.

[12] B. S. Nie and S. M. Duan, "The adsorption essence of gas on coal surface," Journal of Taiyuan University of Technology, vol. 29, no. 4, pp. 417-421, 1998.

[13] Y. J. Cui, Q. L. Zhang, and X. H. Yang, "The absorption properties of different coal and changes of the absorption heating," Natural Gas Industry, vol. 23, no. 4, pp. 130-131, 2003.

[14] D. Zhao, Y. S. Zhao, Z. C. Feng, Z. X. Liu, and T. Liu, "Experiments of methane adsorption on raw coal at $30-270^{\circ} \mathrm{C}$," Energy Sources, Part A: Recovery, Utilization, and Environmental Effects, vol. 34, no. 4, pp. 324-331, 2011.

[15] D. M. Ma, S. A. Zhang, and Y. B. Lin, "Isothermal adsorption and desorption experiment of coal and experimental results accuracy fitting," Journal of China Coal Society, vol. 36, no. 3, pp. 477-480, 2011.

[16] C. O. Karacan, "Assessment of energetic heterogeneity of coals for gas adsorption and its effect on mixture predictions for coalbed methane studies," Fuel, vol. 79, no. 15, pp. 19631974, 2000.

[17] Z. Liu, Q. Zhang, and X. Yang, "The adsorption properties of different coal and changes of the adsorption heating," Natural Gas Industry, vol. 23, no. 4, pp. 130-131, 2003.

[18] X. F. Liu and X. Q. He, "Effect of pore characteristics on coalbed methane adsorption in middle-high rank coals," Adsorption, vol. 23, no. 1, pp. 3-12, 2017.

[19] J. Ibarra, E. Munoz, and R. Moliner, "FTIR study of the evolution of coal structure during the coalification process," Organic Geochemistry, vol. 24, no. 6-7, pp. 725-735, 1996.

[20] P. Y. Hao, Influence of High Pressure with Fracturing Fluid on Microstructure of Low-Medium Rank Coal Reservoir, Taiyuan University of Technology, Taiyuan, 2019.
[21] S. Y. Wang, Study of Lignite Structure by Molecular Dynamics Simulation and Quantum Chemistry, Taiyuan University of Technology, Taiyuan, 2004.

[22] Z. W. Li, Study on Microstructure Characteristics of Low Rank Coal and Its Control Mechanism on Gas Adsorption / Desorption, China University of Mining and Technology, Xuzhou, 2015.

[23] M. Arif, F. Jones, A. Barifcani, and S. Iglauer, "Influence of surface chemistry on interfacial properties of low to high rank coal seams," Fuel, vol. 194, no. 2017, pp. 211-221, 2017.

[24] S. Q. Wang, Y. G. Tang, H. H. Schobert, Y. N. Guo, and Y. Su, "FTIR and13C NMR investigation of coal component of late Permian coals from southern China," Energy \& Fuels, vol. 25, no. 12, pp. 5672-5677, 2011.

[25] J. V. Ibarra, R. Moliner, and A. J. Bonet, "FT-i.r. investigation on char formation during the early stages of coal pyrolysis," Fuel, vol. 73, no. 6, pp. 918-924, 1994.

[26] K. J. Li, R. Khanna, J. L. Zhang et al., "Comprehensive investigation of various structural features of bituminous coals using advanced analytical techniques," Energy \& Fuels, vol. 29, no. 11, pp. 7178-7189, 2015.

[27] Y. T. Guo and M. R. Bustin, "Micro-FTIR spectroscopy of liptinite macerals in coal," International Journal of Coal Geology, vol. 36, no. 3-4, pp. 259-275, 1998.

[28] D. W. Krevelen, Coal: Typology, Physics, Chemistry, Constitution, Elsevier Science, Amsterdam, 1993.

[29] P. F. Li, Pore Structural Characterization and Adsorption Properties of Deep Coal Reservoir Based on Molecular Simulation: A Case Study from Daning-Jixian District Coalbed, Taiyuan University of Technology, Taiyuan, 2019.

[30] Z. Zou, Adsorption Characteristics and Their Thermodynamic Evolution in Variable Rank Coals, China University of Geosciences (Beijing), Beijing, 2020.

[31] Y. Y. Chen, M. Mastalerz, and A. Schimmelmann, "Characterization of chemical functional groups in macerals across different coal ranks via micro-FTIR spectroscopy," International Journal of Coal Geology, vol. 104, pp. 22-33, 2012.

[32] C. H. Liang, W. Q. Liang, and W. Li, "Functional groups of different coal ranks based on infrared spectroscopy," Coal Science and Technology, vol. 48, no. S1, pp. 182-186, 2020.

[33] X. Q. He, X. F. Liu, B. S. Nie, and D. Song, "FTIR and Raman spectroscopy characterization of functional groups in various rank coals," Fuel, vol. 206, no. 2017, pp. 555-563, 2017.

[34] H. J. Li, Study on Thermal Effect of Methane Adsorption in Coal, China University of Mining and Technology, Xuzhou, 2019.

[35] Z. W. Li, B. Q. Lin, Z. Y. Hao, Y. B. Gao, and F. F. Liu, "Fractal characteristics of porosity for porous media in coal mass," Journal of Mining \& Safety Engineering, vol. 216, 2021. 